\title{
Equilibrium spin-glass transition of magnetic dipoles with random anisotropy axes on a site-diluted lattice
}

\author{
Julio F. Fernández ${ }^{1, *}$ and Juan J. Alonso ${ }^{2}$ \\ ${ }^{1}$ Instituto de Ciencia de Materiales de Aragón, CSIC-Universidad de Zaragoza, 50009 Zaragoza, Spain \\ ${ }^{2}$ Física Aplicada I, Universidad de Málaga, 29071 Málaga, Spain \\ (Received 29 December 2008; revised manuscript received 19 May 2009; published 17 June 2009)
}

\begin{abstract}
We study partially occupied lattice systems of classical magnetic dipoles which point along randomly oriented axes. Only dipolar interactions are taken into account. The aim of the model is to mimic collective effects in disordered assemblies of magnetic nanoparticles. From tempered Monte Carlo simulations, we obtain the following equilibrium results. The zero-temperature entropy approximately vanishes. Below a temperature $T_{c}$, given by $k_{B} T_{c}=(0.95 \pm 0.1) x \varepsilon_{d}$, where $\varepsilon_{d}$ is a nearest-neighbor dipole-dipole interaction energy and $x$ is the site occupancy rate, we find a spin-glass phase. In it, (1) the mean value $\langle|q|\rangle$, where $q$ is the spin overlap, decreases algebraically with system size $N$ as $N$ increases, and (2) $\delta|q| \simeq 0.5\langle|q|\rangle \sqrt{T / x}$, independently of $N$, where $\delta|q|$ is the root-mean-square deviation of $|q|$.
\end{abstract}

DOI: 10.1103/PhysRevB.79.214424

PACS number(s): 75.10.Nr, 75.10.Hk, 75.40.Mg

\section{INTRODUCTION}

Magnetic dipole-dipole interactions play a fundamental role in magnetic phenomena. Their long-range nature gives rise to magnetic domains in ferromagnets. On the other hand, because of their weak strength, they usually play an insignificant role in determining the Curie temperatures and in critical phenomena of atomic crystals. In assemblies of magnetic nanoparticles (NPs), things can be very different. Because ferromagnetic NPs of up to thousands of Bohr magnetons have a single magnetic domain, ${ }^{1}$ dipole-dipole interactions among such NPs can be very large and can thus dominate their collective behavior. ${ }^{2}$ Often, both the orientation of the crystallites of which NPs are made as well as their positions are disordered in the assembly, and therefore behave much as a system of interacting magnetic dipoles with randomly oriented magnetic easy axes. These systems of random axes dipoles (RADs) are clearly frustrated, since two different dipoles give rise to magnetic fields at any given point which are not in general collinear. The sort of timedependent behavior that is expected of spin glasses has been observed in experiments ${ }^{3-5}$ as well as in simulations ${ }^{6-10}$ of disordered assemblies of magnetic NPs. Because these systems evolve in time very slowly (exhibiting aging ${ }^{3,6}$ and other memory ${ }^{11}$ effects), evidence for a thermodynamic spinglass phase in them is more difficult to come by. One of us has recently given numerical evidence for the existence of an equilibrium spin-glass phase for a fully occupied lattice of dipoles with randomly oriented axes. ${ }^{12}$ On the other hand, numerical evidence against such a phase has been given for site-diluted lattice systems of magnetic dipoles with parallel axes. $^{13}$

One might expect site-diluted systems of RADs to behave as fully occupied ones, and therefore to have equilibrium spin-glass phases at low temperatures. For support of this expectation, consider rescaling distances in a dilute system of RADs. Because dipole-dipole interactions decay with distance $r$ as $r^{-3}$, letting $r \rightarrow b r$ merely redefines dipole-dipole interaction energies as $\varepsilon_{d} \rightarrow b^{-3} \varepsilon_{d}$. This would imply all physical quantities for systems of RADs in three dimensions
(3D) with different values of site concentration $x$ collapse onto the same curve when plotted versus $T / x$, where $T$ is the temperature. This argument holds for randomly located RADs on a continuous space in 3D. It is therefore expected to hold approximately for a site-diluted lattice if $x \ll 1$, but not necessarily for lattices with higher concentrations of RADs. This is why dipoles with parallel axes can have an antiferromagnetic phase at low temperature on a fully occupied simple cubic lattice, ${ }^{14}$ but appear to have no condensed phase of any sort on a very dilute lattice. ${ }^{13}$ Similarly, the existence of a spin-glass phase for RADs for $x<1$ does not follow from its existence for $x=1$.

Our main aim here is to find, by means of the parallel tempered Monte Carlo (TMC) algorithm, ${ }^{15,16}$ whether an equilibrium spin-glass phase exists in a site-diluted system of RADs in 3D. We also aim to establish, if the spin-glass phase does exist, whether in the condensed phase (1) there is a single extended state, ${ }^{17}$ as in a ferromagnet or in the droplet model of spin glasses, ${ }^{18}$ or (2) there are multiple extended states, as in the $X Y$ model in two dimensions (2D) (Ref. 19) or in the replica symmetry breaking theory of spin glasses. ${ }^{20}$

The paper is planned as follows. In Sec. II we specify the RAD model and describe how we apply the parallel TMC algorithm. ${ }^{15,16} \mathrm{We}$ do this for $x=0.35$ and $x=0.5$. Results are given in Sec. III. The entropy $S$ follows from our data for the specific heat by numerical integration. It approximately vanishes at zero temperature. ${ }^{21}$ More precisely, $S<0.01 k_{B}$ at $T$ $=0$. We provide numerical evidence for the existence of an equilibrium spin-glass phase below a transition temperature $T_{c}$. The evidence comes from the behavior of the distribution of the spin overlap parameter $q .^{22,23}$ In the spin-glass phase, $\left\langle q^{2}\right\rangle$ seems to vanish in the macroscopic limit, but only as a power of system size, in accordance with the existence of quasi-long-range order. For both $x=0.35$ and $x=0.5, k_{B} T_{c}$ $=(0.95 \pm 0.1) x \varepsilon_{d}$, where $\varepsilon_{d}$ is a nearest-neighbor dipoledipole interaction energy which is defined in Sec. II. (Within errors, the value of $T_{c}$ for $x=1$ we have previously obtained ${ }^{12}$ also satisfies this expression.) For $T \leqq 0.9 T_{c}, \delta|q|$, that is, the root-mean-square deviation of $|q|$, fulfills $\delta|q|$ $\simeq 0.5\langle|q|\rangle \sqrt{T / T_{c}}$, independently of system size. This result is 
compared with its counterpart for the $X Y$ model in 2D. Results are discussed in Sec. IV.

\section{MODEL AND METHOD}

We treat systems of magnetic dipoles on simple cubic lattices. We place a dipole on each lattice site with probability $x$ and leave the site unoccupied with probability $1-x$. All dipoles point along randomly oriented anisotropy axes. With this model we aim to mimic assemblies of NPs in which uniaxial anisotropy energies are an order of magnitude larger than the largest dipole-dipole energy. Then, anisotropy energy barriers are not so large as to freeze spin reorientations near the spin-glass temperature, but are sufficiently large to restrict spins to point "up" or "down" approximately along the easy magnetization axes. We term this the random-axesdipolar (RAD) model. The Hamiltonian is given by

$$
\mathcal{H}=\frac{1}{2} \sum_{i j} \sum_{\alpha \beta} T_{i j}^{\alpha \beta} S_{i}^{\alpha} S_{j}^{\beta},
$$

where the first sum is over all occupied sites $i$ and $j$ of a simple cubic lattice, $S_{i}^{\alpha}$ is the $\alpha$ component of a classical three-component spin on site $i$,

$$
T_{i j}^{\alpha \beta}=\varepsilon_{d}\left(a / r_{i j}\right)^{3}\left(\delta_{\alpha \beta}-3 r_{i j}^{\alpha} r_{i j}^{\beta} / r_{i j}^{2}\right),
$$

$r_{i j}$ is the distance between $i$ and $j, \varepsilon_{d}$ is an energy, and $a$ is a nearest-neighbor distance. Each spin points along a randomly chosen direction. These equations can be cast into a form that is manifestly Ising type by letting $\hat{\mathbf{u}}_{j}$ be (1) a null vector if the $j$ site is unoccupied and (2) a three-component unit vector chosen randomly from a spherically uniform distribution if the $j$ site is occupied, and defining a pseudospin $\sigma_{j}= \pm 1$ for each site, such that $\mathbf{S}_{j}=\hat{\mathbf{u}}_{j} \sigma_{j}$. We can then write

$$
\mathcal{H}=-\frac{1}{2} \sum_{i j} J_{i j} \sigma_{i} \sigma_{j},
$$

where $J_{i j}=-\Sigma_{\alpha, \beta} T_{i j}^{\alpha \beta} u_{i}^{\alpha} u_{j}^{\beta}$. Thus, the RAD model is an Ising model whose bonds $J_{i j}$ are determined by the dipole-dipole terms $T_{i j}^{\alpha \beta}$ and the set of three-component randomly oriented unit vectors $\left\{\hat{\mathbf{u}}_{j}\right\}$.

From here on, unless we state otherwise, we let $k_{B}=1$, where $k_{B}$ is Boltzmann's constant and give all temperatures in terms of $\varepsilon_{d}$. We use periodic boundary conditions. Details and justification are given in Refs. 12, 14, 16, and 24.

In order to arrive at equilibrium results, we make use of the parallel TMC algorithm. ${ }^{15}$ This enables one to circumvent large energy barriers that can trap a system's state. We apply the TMC algorithm as follows. We run (in parallel) several identical systems at different temperatures: a system at temperature $T_{0}$, a second one at $T_{0}+\Delta T$, and so on, at equally spaced temperatures, up to $T_{m}$. We choose $T_{m}$ to be at least twice as large as what we expect to be the transition temperature between the paramagnetic and spin-glass phases. We let each system evolve under the Metropolis MC algorithm for $10 \mathrm{MC}$ sweeps before pairs of systems are given a chance to exchange their states. More specifically, pairs of systems at temperatures $\left(T_{0}\right.$ and $\left.T_{0}+\Delta T\right),\left(T_{0}+2 \Delta T\right.$ and $T_{0}$

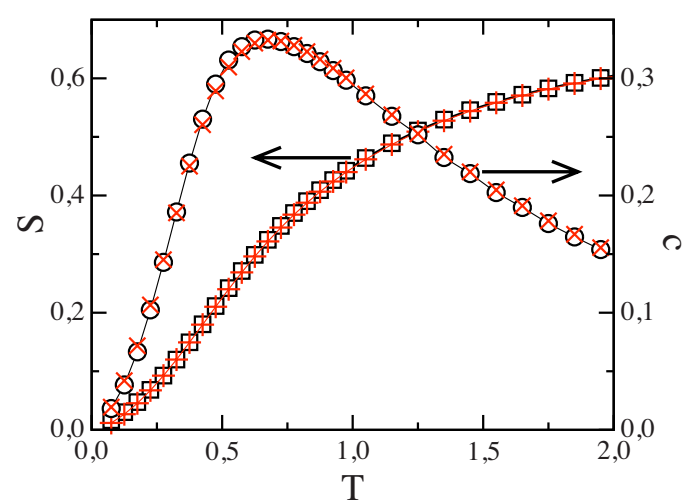

FIG. 1. (a) (Color online) Plots of $c$ and of $S$ vs $T$, for $x=0.35$. Data points (black) $\bigcirc$ (for $N=605$ ) and (red) $\times$ (for $N=179$ ) are for the specific heat, while (black) $\square$ (for $N=605$ ) and (red) + (for $N$ $=179$ ) are for the entropy. Errors, $\delta S$, for $S$ increase as $T$ decreases, from $\delta S \approx 0.002$ for $T=4$ to $\delta S \approx 0.004$ for $T<0.1$.

$+3 \Delta T)$, and so on, are given a chance to exchange states every $10 \mathrm{MC}$ sweeps, and pairs of systems at temperatures $\left(T_{0}+\Delta T\right.$ and $\left.T_{0}+2 \Delta T\right),\left(T_{0}+3 \Delta T\right.$ and $\left.T_{0}+4 \Delta T\right)$, and so on, are given a chance to exchange states at times in between. Under TMC rules, these chances are as follows. A system at temperature $T$ that is in state 2 , and another one at $T+\Delta T$ in state 1 , exchange states with probability $P=1$ if $E_{1}<E_{2}$ and $\Delta T>0$, but $P=\exp (-\Delta \beta \delta E)$, where $\Delta \beta=1 / T-1 /(T+\Delta T)$ and $\delta E=E_{1}-E_{2}$, if $E_{1}>E_{2}$ and $\Delta T>0 .{ }^{16}$

A sufficiently small value of $\Delta T$ must be chosen in order to keep $\exp (-\Delta \beta \delta E)$ from becoming too small. This will often be fulfilled if $\Delta \beta \Delta E \lesssim 1$, where $\Delta E$ is the mean energy difference between two systems at temperatures $T$ and $T$ $+\Delta T$. The required condition, $\Delta T \lesssim T / \sqrt{N c}$, where $N$ is the number of dipoles in the system, follows for $\Delta T$ if we replace $\Delta E$ by $c N \Delta T$ (we thus define $c$ as the specific heat per spin). From plots of the specific heat vs $T$, such as the one shown in Fig. 1 for systems of 179 and of 605 magnetic dipoles on lattices with 0.35 of their sites occupied, one can get upper bounds for $\Delta T$. (How the data points shown in Fig. 1 were obtained is explained in Sec. III.) Values of $\Delta T$ as well as of other parameters for all TMC runs are given in Table I.

In order to probe for spin-glass behavior, we define, as is usual, the spin overlap parameter. ${ }^{22,23}$ First, let

$$
\phi_{j}=\sigma_{j}^{(1)} \sigma_{j}^{(2)},
$$

where $\sigma_{j}^{(1)}$ and $\sigma_{j}^{(2)}$ are the pseudospins [defined above Eq. (3)] on site $j$ of identical twin replicas 1 and 2 of the system. Clearly,

$$
q=N^{-1} \sum_{j} \phi_{j}
$$

is a measure of the spin configuration overlap between replicas 1 and 2. Thus, $|q|=1$ if either $\sigma_{j}^{(1)}=\sigma_{j}^{(2)}$ for all $j$ or $\sigma_{j}^{(1)}=-\sigma_{j}^{(2)}$ for all $j$. We also define the moments of $q, q_{k}$ $=\left\langle|q|^{k}\right\rangle$, for $k=1,2$, and 4 , where $\langle\ldots\rangle$ stands for an average which we next specify. 
TABLE I. Simulation parameters. $x$ is the probability that any given site is occupied by a magnetic dipole; $N$ is the mean number of magnetic dipoles in the system; $\Delta T$ is the temperature step in the TMC runs; $T_{m}$ is the highest temperature of all systems; $N_{s}$ is the number of disordered system pairs; MCS is the number of tempered Monte Carlo sweeps.

\begin{tabular}{lcccccccc}
\hline \hline$x$ & 0.35 & 0.35 & 0.35 & 0.35 & 0.5 & 0.5 & 0.5 & 0.5 \\
$N$ & 22.4 & 75.6 & 179.2 & 604.8 & 32 & 108 & 256 & 864 \\
$\Delta T$ & 0.05 & 0.05 & 0.05 & 0.05 & 0.05 & 0.05 & 0.05 & 0.05 \\
$T_{m}$ & 1.0 & 1.0 & 1.0 & 1.0 & 1.3 & 1.3 & 1.3 & 1.3 \\
$N_{s}$ & 10000 & 3000 & 1250 & 900 & 5000 & 3000 & 1500 & 150,600 \\
$\mathrm{MCS}$ & 50000 & $10^{5}$ & $5 \times 10^{6}$ & $10^{6}$ & 50000 & 50000 & $10^{6}$ & $10^{6}, 10^{5}$ \\
\hline \hline
\end{tabular}

Suppose our TMC runs last for a time $t$, and assume that, within the temperature range we are interested in, equilibration takes place in a time less than $t / 2$. During the TMC runs, we write, at equally spaced time intervals within the $(t / 2, t)$ range, the spin configuration for each temperature. We later draw from these written configurations the numbers for $\sigma_{j}^{(1)}$ that go into Eq. (4). This procedure is repeated for the same system (that is, for a system with the same anisotropy axes), but starting from different initial conditions. From this second set of configurations, we draw the numbers for $\sigma_{j}^{(2)}$ that go into Eq. (4). A value of $q$ for each time $t$ and temperature $T$ is thus obtained from Eq. (5). We first average all quantities of interest over $t$ and finally repeat these pairs of TMC runs a number $N_{s}$ of times for different realizations of axes orientations, from which the average values we are reporting are obtained. Values we have used in our TMC runs for $N_{s}$ and for $t$ (labeled MCS therein) are given in Table I.

Finally, in order to check that equilibration actually takes place as assumed, we also calculate the spin overlap $\tilde{q}$, not between identical twin replicas, but between spin configurations at two different times $t_{0}$ and $t_{1}$ of the same TMC run. We do this for several values of $t_{0}$ and $t_{1}$ in the neighborhoods of $t / 2$ and $t$, respectively, and average over different random axes realizations, in order to obtain $\tilde{q}_{1}$. For $t$ sufficiently large, $\tilde{q}_{1}=q_{1}$. We find this is fulfilled for all parameter values given in Table I, except for (1) $x=0.35, N=604.8$, and $T \leqq 0.2$, and (2) for $x=0.5, N=864$, and $T \leqq 0.35$.

\section{RESULTS}

Plots of the specific heat and of the entropy vs $T$ for the RAD model are shown in Fig. 1 for $x=0.35$. The data for the specific heat follow from numerical derivatives of the energy with respect to $T$. We obtain $S$ from $S(T)=\ln 2$ $+\int_{\infty}^{T} c\left(T^{\prime}\right) / T^{\prime} d T^{\prime}$. Our data cover the temperature range (not all of it shown in Fig. 1) $0.05<T<4$. For a numerical integration, we must extrapolate our data for $c(T)$ beyond $T=4$. To this end, we use the leading term of an energy expansion in powers of $1 / T$, which gives $c(T) \rightarrow A / T^{2}$. A fit of the value of $A$ to our data for $c(T)$ at $T=4$ leads to a $\Delta S \simeq 0.027$ contribution to the entropy from the $T>4$ range. We thus obtain $S(T)$. It is exhibited in Fig. 1. Errors for $S(T)$ come in two approximately equal pieces: (1) an error of roughly 0.002 from the $4<T<\infty$ range and (2) an error of roughly 0.002 , from errors in the data for the specific, which enter the integral $\int_{\infty}^{T} c\left(T^{\prime}\right) / T^{\prime} d T^{\prime}$. We finally obtain $S=0.015 \pm 0.004$ for
$T=0.05$, and extrapolations below $T=0.05$ yield $S<0.01$ at $T=0$. Similarly, we find $S<0.01$ at $T=0$ for $x=0.50$.

Our results for $q_{1}$ and $q_{2}$ follow. Note we use an absolute value in the definition of $q_{1}$. Recall that $N q_{2}=O(1)$ in the paramagnetic phase and diverges at the transition temperature $T_{c}$. Above the lower critical dimension $d_{c}, q_{2}=O(1)$ in the droplet model of the spin-glass phase. ${ }^{18}$ Our data show that $q_{2}$ decreases as $N$ increases, for all nonzero temperatures. Log-log plots of $q_{2}$ vs $N$ are exhibited in Figs. 2(a) and 2(b) for various values of $T$. The behavior of $q_{2}$ for $T \leqq 0.3$ and $T \leqq 0.45$ for $x=0.35$ and $x=0.5$, respectively, is consistent with $q_{2} \sim N^{-y}$, where $y$ is some positive parameter that depends on $T$. This behavior is reminiscent of the $X Y$ model in $2 \mathrm{D} .{ }^{19}$ For higher values of $T, q_{2}$ vs $N$ clearly curves downward, in accordance with a faster than algebraic in $N$ decay, as one expects for the paramagnetic phase. Log-log plots of $q_{1}^{2}$ are also shown in Figs. 2(a) and 2(b) for various values of $T$. Note that $q_{2} / q_{1}^{2}>1$ for all $N$ and nonzero $T$, which implies a nonvanishing uncertainty in $\left|q_{r}\right|$, where $q_{r}=q / q_{1}$, for $T>0$.

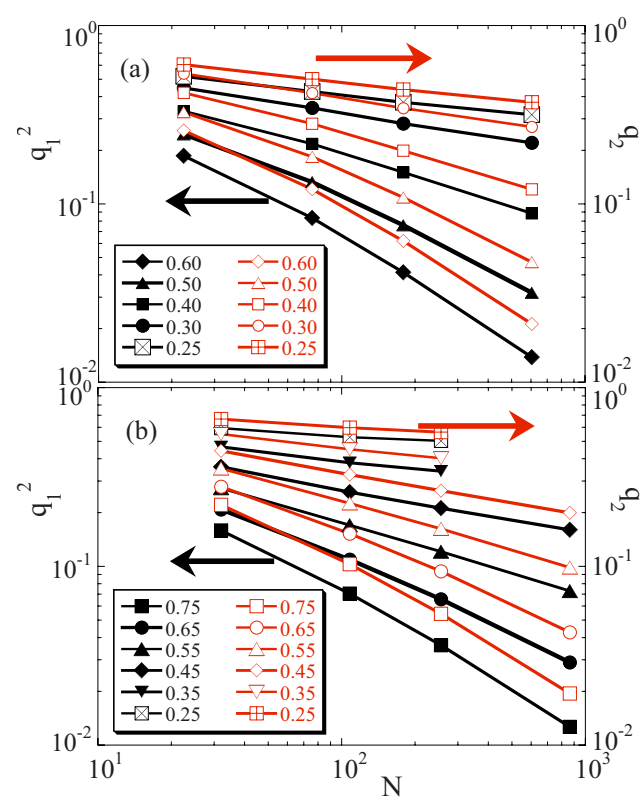

FIG. 2. (Color online) (a) Log-log plots of $q_{1}$ and of $q_{2}$ vs $N$ for $x=0.35$ and the shown values of $T$. Lines are guides for the eyes. (b) Same as in (a) but for $x=0.5$. Some data points for small $T$ and large $N$ are missing because our TMC runs did not reach equilibration for them. 


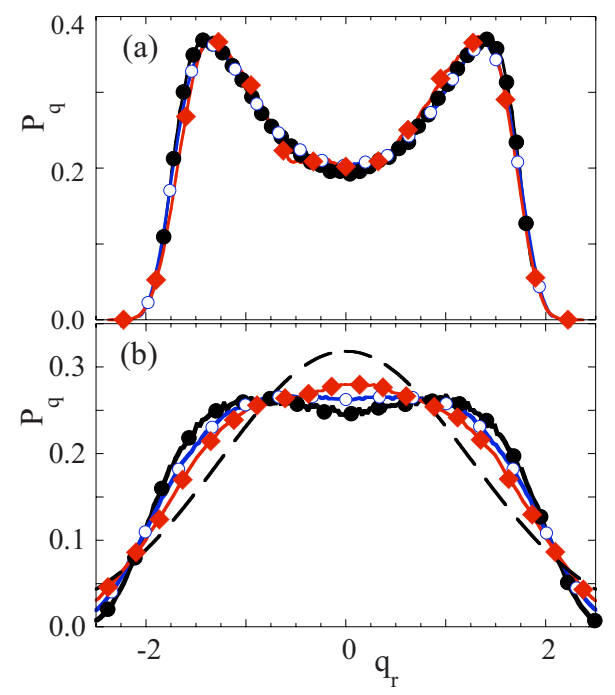

FIG. 3. (Color online) (a) Plots of the probability $P_{q}$ vs $q_{r}$, for $x=0.50$ and $T=0.45$.,$\bigcirc$, and $\diamond$ are for $N=108,256$, and 864 , respectively. Lines are guides for the eyes. For clarity, only $30 \%$ of the data points are shown. (b) Same as in (a) but for $T=0.60$. For comparison, a dashed line is shown for $(1 / \pi) \exp \left(-q_{r}^{2} / \pi\right)$, which ensues for a macroscopic paramagnetic phase.

We next report results for the probability distribution $P_{q}\left(q_{r}\right)$. Unless stated otherwise, all data given below are for a normalized $P_{q}\left(q_{r}\right)$, that is, $\int P_{q}\left(q_{r}\right) d q_{r}=1$. Recall that in the paramagnetic phase, because spin-spin correlation lengths are finite, the central limit theorem implies $q_{r}$ is normally distributed for macroscopic systems. Plots of $P_{q}$ vs $q_{r}$ are shown in Fig. 3(a) (for $T=0.45$ ) and Fig. 3(b) (for $T=0.60$ ), both for $x=0.50$. The distribution of $q$ appears to be size independent for $T=0.45$. For $T=0.60$, on the other hand, $P_{q}\left(q_{r}\right)$ drifts with system size. The drift seems consistent

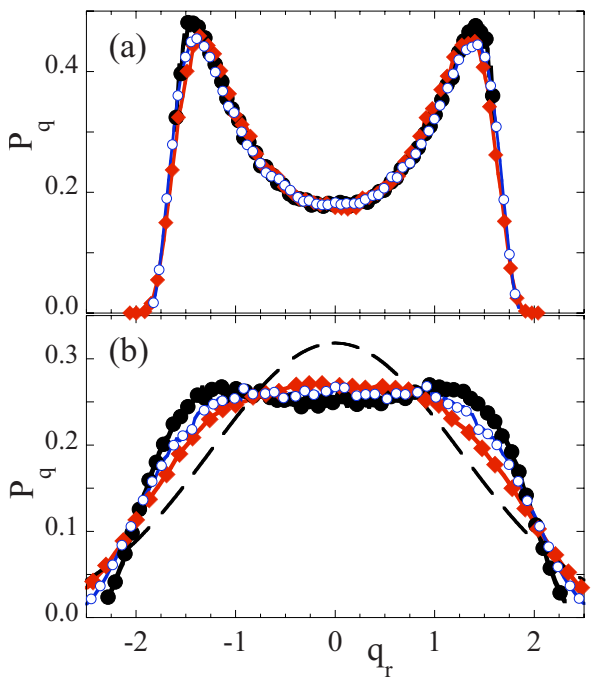

FIG. 4. (Color online) (a) Plots of the probability distribution $P_{q}$ vs $q_{r}$, for $x=0.35$ and $T=0.3$.,$\bigcirc$, and $\bullet$ are for $N=76,179$, and 605 , respectively. Lines are guides for the eyes. For clarity, only $30 \%$ of the data points are shown. (b) Same as in (a) but for $T=0.45$. For comparison, a dashed line is shown for $(1 / \pi)$ $\times \exp \left(-q_{r}^{2} / \pi\right)$, which ensues for a macroscopic paramagnetic phase. with $P_{q}\left(q_{r}\right) \rightarrow(1 / \pi) \exp \left(-q_{r}^{2} / \pi\right)$ as $N \rightarrow \infty$, which would be in accordance with a paramagnetic phase. Similar remarks apply to the plots exhibited in Fig. 4(a) (for $T=0.30$ ) and Fig. 4(b) (for $T=0.45$ ), both for $x=0.35$. Clearly, $0.30<T_{c}$ $<0.45$ for $x=0.35$, and $0.45<T_{c}<0.60$ for $x=0.50$. For $T$ $<T_{c}$, plots of $P_{q}$ vs $q_{r}$ (not shown) become sharply peaked only as $T \rightarrow 0$. Now, if it turns out that $d_{c}=3$ for systems of RADs, then $P_{q}\left(q_{r}\right)$ ought to exhibit critical behavior for all $T \leq T_{c}$, more specifically, $P_{q}\left(q_{r}\right)$ ought to be size independent. This sort of behavior is best summarized by the mean square deviation of $\left|q_{r}\right|, \Delta_{q}^{2} \equiv\left\langle q_{r}^{2}\right\rangle-\left\langle\left|q_{r}\right|\right\rangle^{2}$.

Plots of $\Delta_{q}^{2}$ vs $T / x$ are shown in Fig. 5(a) (for $x=0.35$ ) and in Fig. 5(b) for $x=0.50$ for various values of $N$. Clearly, curves in Figs. 5(a) and 5(b) differ only slightly, as expected from the argument given in Sec. I, that variations in $T$ and $x$ have an effect only through $T / x$ if $x \ll 1$. The data points in both figures suggest $\Delta_{q}^{2} \rightarrow \pi / 2-1$ as $N \rightarrow \infty$, for $T / x \geqslant 1$. This is expected for a macroscopic paramagnet, since $P_{q}\left(q_{r}\right)$ is a normal distribution then. For $T / x \leqq 0.8$,

$$
\Delta_{q} \simeq 0.5 \sqrt{T / x}
$$

provides the best fit to the data points shown in Figs. 5(a) and $5(\mathrm{~b})$. This is in contrast with the behavior, $\Delta_{q}^{2} \rightarrow 0$ as

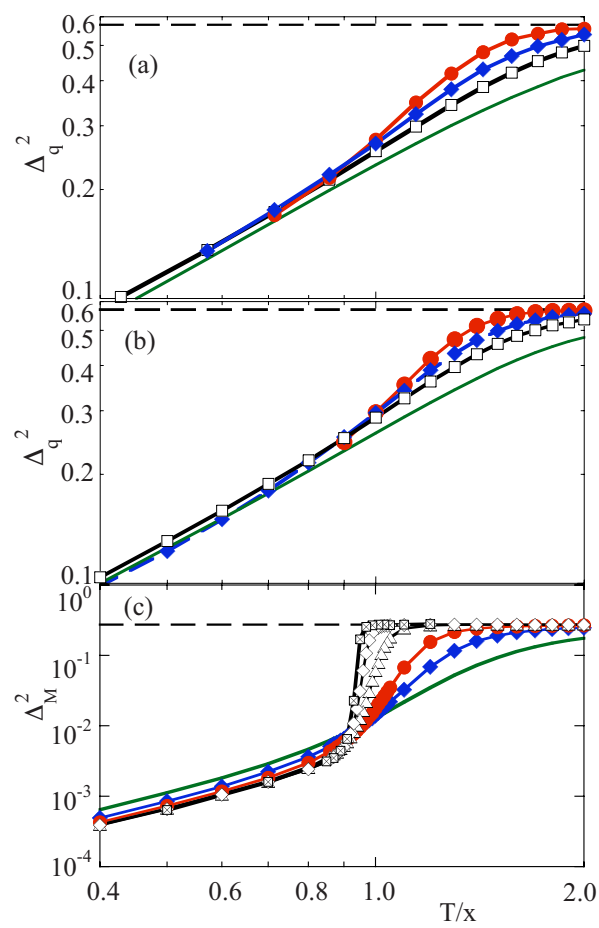

FIG. 5. (Color online) (a) Plots of $\Delta_{q}^{2}$ vs $T$ for $x=0.35$. (red) (blue) $\bullet$, and (black) $\square$ are for $N=604.8,179.2$, and 75.6, respectively. The continuous (green) line is for $N=22.4$. The dashed line is for macroscopic paramagnet. (b) Same as in (a) but for $x=0.50$. (red), (blue), and $\square$ (black) are for $N=864,256$, and 108, respectively. The continuous (green) line is for $N=32$, and the dashed line is for $\pi / 2-1$. (c) Same as in (a), but for the mean square relative deviation $\Delta_{M}^{2}$ of $|M|$, where $M$ is the magnetic moment of $X Y$ systems of $L \times L$ spins in $2 \mathrm{D}$ vs $T / x$, where $x=1$, for $L=4$ (green line), $L=8$ (blue $\diamond), L=16$ (red $\bullet$ ), $L=64$ (black $\triangle$ ), $L$ $=256($ black $\diamond)$, and $L=1024$ (black $\otimes)$. 


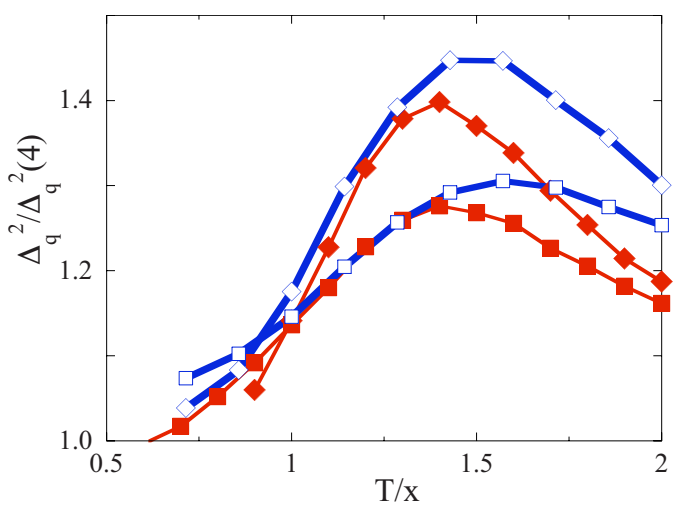

FIG. 6. (Color online) Plots of $\Delta_{q}^{2} / \Delta_{q}^{2}(L=4)$ vs $T$, where $\Delta_{q}^{2}(L$ $=4)$ is $\Delta_{q}^{2}$ for $L=4$. All ( ) are for $x=0.35$ and $L=12$, and all (ם) are for $x=0.35$ and $L=8$. All $(\diamond)$ are for $x=0.50$ and $L=12$, and all ( $\square$ ) are for $x=0.50$ and $L=8$. Lines are guides for the eyes.

$N \rightarrow \infty$, that one expects for the ordered phase of ferromagnets such as the Ising model in two or higher dimensions. For a more relevant comparison, we show in Fig. 5(c) plots of the mean square relative deviation $\Delta_{M}^{2}$ of $|M|$, where $M$ is the magnetic moment, of the $X Y$ model in 2D. Notice there is no counterpart in Figs. 5(a) and 5(b) for the data points shown for rather large systems in Fig. 5(c).

Now, it is hard to see in Figs. 5(a) and 5(b) where curves merge or cross, near $T / x=0.9$. In order to enhance the differences between $\Delta_{q}^{2}$ curves for different values of $L$, we plot $\Delta_{q}^{2} / \Delta_{q}^{2}(L=4)$ vs $T / x$. These plots are shown in Fig. 6 for $x$ $=0.35$ and $x=0.50$. The weak dependence on $x$ is remarkable. Notice that whereas curves for $L=12$ and $L=8$ cross near $T / x=0.95$ for $x=0.35$ and for $x=0.50$, the same curves cross the $\Delta_{q}^{2} / \Delta_{q}^{2}(L=4)=1$ horizontal line only, if at all, for much smaller values of $T / x$. It thus appears that curves for $\Delta_{q}^{2}$ increasingly larger system sizes merge or cross at increasingly larger values of $T / x$.

We show plots of $P_{q}(0)$ vs $N$ for $x=0.35$ and various temperatures in Fig. 7. In the paramagnetic phase, we expect $P_{q}(0) \rightarrow 1 / \pi$ as $N \rightarrow \infty$, as follows from a normal distribution of $q_{r}$ about $q_{r}=0$. Now, for $T / x \geq 1.0$, we see that $P_{q}(0)$ $<1 / \pi$, but $P_{q}(0)$ increases as $N$ increases. On the other hand, for $T / x \leq 0.857, P_{q}(0)$ remains, within errors, constant. This is again in contrast with the behavior, $P_{q}(0) \rightarrow 0$ as $N$ $\rightarrow \infty$, that one expects for the ordered phase of ferromagnets such as the Ising model in two or higher dimensions, but is in accordance with $d_{c} \simeq 3$. Finally, taking into account all our observations above, we arrive at our best estimate for $T_{c}$ : $T_{c} / x=0.95 \pm 0.1$.

\section{DISCUSSION}

By tempered Monte Carlo calculations, we have studied systems of RADs in simple cubic lattices in which each site is occupied by a magnetic dipole with probability $x$. Systems sizes, Monte Carlo run lengths, and other details about the calculations can be found in Table I. The entropy $S$ as a function of temperature, which follows from our data for the specific heat, is shown in Fig. 1 for $x=0.35$. $S$ approximately vanishes at zero temperature. More precisely, $S<0.01 k_{B}$ at

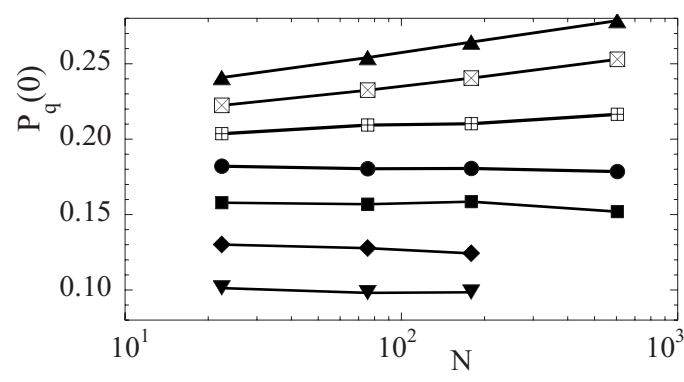

FIG. 7. Plots of $P_{q}(q=0)$ vs $N$, for $x=0.35$, for $T / x=1.286(\boldsymbol{\Delta})$, $T / x=1.143(\otimes) T / x=1.0(\boxplus), T / x=0.857(\bullet), T / x=0.714(\mathbf{\square})$, $T / x=0.57(\diamond)$, and $T / x=0.429,(\nabla)$.

$T=0$ for both $x=0.35$ and $x=0.50 .{ }^{21}$ Note also that a vanishing zero-temperature entropy implies that trapping above the lowest energy states as $T \rightarrow 0$ occurs only very rarely in our TMC runs.

We have obtained the spin overlap parameter defined in Eq. (5). More specifically, we have obtained the mean value of $|q|$, which we term $q_{1}$, and the mean square value of $q$, which is $q_{2}$. The plots in Fig. 2(a) (for $x=0.35$ ) and in Fig. 2(b) (for $x=0.50$ ) suggest both $q_{1}$ and $q_{2}$ vanish for all nonzero $T$ as $N \rightarrow \infty$. As can be gathered from those plots, both $q_{1}^{2} \sim N^{-y}$ and $q_{2} \sim N^{-y}$, where $y$ is some positive parameter that depends on $T$, for $k_{B} T / x \leqq 0.9 \varepsilon_{d}$. This points to quasilong-range order. It is reminiscent of the $X Y$ model in $2 \mathrm{D}$ and suggests the lower critical dimension $d_{c}$ of the RAD model is at or near 3D. However, we have no grounds for taking the analogy between the $X Y$ model in 2D and the RAD model in $3 \mathrm{D}$ any further than this. We make no claim on the relation between the universality classes these systems may belong to.

We have also studied the probability distribution $P_{q}\left(q_{r}\right)$, where $q_{r}=q / q_{1}$. Within errors, $P_{q}\left(q_{r}\right)$ is independent of system size at $T=T_{c}$, as is illustrated in Figs. 4(a) and 3(a) for $k_{B} T / x \simeq 0.9 \varepsilon_{d}$. We have also found (not shown) $P_{q}\left(q_{r}\right)$ to be independent of system size at lower temperatures. The results shown in Figs. 5(a) and 5(b) and in Eq. (6), for the mean square deviation of $\left|q_{r}\right|, \Delta_{q}^{2}$, are consistent with a $P_{q}\left(q_{r}\right)$ that is independent of system size for all $k_{B} T / x$ $\lessgtr 0.9 \varepsilon_{d}$. Again, this points to $d_{c} \simeq 3$ for the RAD model and is in contradistinction to results for the nearest-neighbor random bond Ising model in 3D. ${ }^{25}$

\section{ACKNOWLEDGMENTS}

The Instituto Carlos I (at Universidad de Granada) and the BIFI Institute (at Universidad de Zaragoza) have generously allowed us to run on many of their computer cluster nodes. We acknowledge financial support through Grant No. FIS2006-00708 from the Ministerio de Ciencia e Innovación of Spain and from Diputación General de Aragón through Grant No. IMANA-E34. 
*jefe@unizar.es

${ }^{1}$ For a theory of single-domain particle size, see E. H. Frei, S. Shtrikman, and D. Treves, Phys. Rev. 106, 446 (1957); J. R. Childress, C. L. Chen, and M. Natham, Appl. Phys. Lett. 56, 95 (1990); S. Chakraverty and M. Bandyopadhyay, J. Phys.: Condens. Matter 19, 216201 (2007).

${ }^{2}$ S. A. Majetich and M. Sachan, J. Phys. D 39, R407 (2006).

${ }^{3}$ W. Luo, S. R. Nagel, T. F. Rosenbaum, and R. E. Rosensweig, Phys. Rev. Lett. 67, 2721 (1991); T. Jonsson, J. Mattsson, C. Djurberg, F. A. Khan, P. Nordblad, and P. Svedlindh, ibid. 75, 4138 (1995); C. Djurberg, P. Svedlindh, P. Nordblad, M. F. Hansen, F. Bødker, and S. Mørup, ibid. 79, 5154 (1997); T. Jonsson, P. Nordblad, and P. Svedlindh, Phys. Rev. B 57, 497 (1998); H. Mamiya, I. Nakatani, and T. Furubayashi, Phys. Rev. Lett. 82, 4332 (1999).

${ }^{4}$ F. Bert, V. Dupuis, E. Vincent, J. Hammann, and J.-P. Bouchaud, Phys. Rev. Lett. 92, 167203 (2004).

${ }^{5}$ V. S. Zotev, G. F. Rodriguez, G. G. Kenning, R. Orbach, E. Vincent, and J. Hammann, Phys. Rev. B 67, 184422 (2003); G. G. Kenning, G. F. Rodriguez, and R. Orbach, Phys. Rev. Lett. 97, 057201 (2006).

${ }^{6}$ J. O. Andersson, C. Djurberg, T. Jonsson, P. Svedlindh, and P. Nordblad, Phys. Rev. B 56, 13983 (1997).

${ }^{7}$ J. García-Otero, M. Porto, J. Rivas, and A. Bunde, Phys. Rev. Lett. 84, 167 (2000).

${ }^{8}$ M. Ulrich, J. García-Otero, J. Rivas, and A. Bunde, Phys. Rev. B 67, 024416 (2003).

${ }^{9}$ O. Iglesias and A. Labarta, Phys. Rev. B 70, 144401 (2004).

${ }^{10}$ S. Russ and A. Bunde, Phys. Rev. B 75, 174445 (2007).

${ }^{11}$ Memory effects in disordered assemblies of magnetic nanoparticles have been reported in Y. Sun, M. B. Salamon, K. Garnier, and R. S. Averback, Phys. Rev. Lett. 91, 167206 (2003).

${ }^{12}$ J. F. Fernández, Phys. Rev. B 78, 064404 (2008).

${ }^{13}$ J. Snider and C. C. Yu, Phys. Rev. B 72, 214203 (2005).

${ }^{14}$ See, for instance, J. F. Fernández and J. J. Alonso, Phys. Rev. B 62, 53 (2000).

${ }^{15}$ K. Hukushima and K. Nemoto, J. Phys. Soc. Jpn. 65, 1604 (1996); E. Marinari, Advances in Computer Simulation, edited by J. Kerte'sz and Imre Kondor (Springer-Verlag, Berlin, 1998), p. 50; arXiv:cond-mat/9612010.

${ }^{16} \mathrm{~A}$ short justification for the TMC rule can be found in J. F. Fernández and J. J. Alonso, Proceedings of Modeling and Simulation of New Materials: Tenth Granada Lectures, AIP Conference Proceedings Vol. 1091, edited by J. Marro, P. L. Garrido, and P. I. Hurtado (AIP, New York, 2009), pp. 151-161.

${ }^{17} \mathrm{By}$ a single extended state we mean that the spin overlap parameter (defined in Sec. III) can take a single value. For further details, see J. Sinova, G. Canright, H. E. Castillo, and A. H. MacDonald, Phys. Rev. B 63, 104427 (2001).

${ }^{18}$ D. S. Fisher and D. A. Huse, J. Phys. A 20, L1005 (1987); D. A. Huse and D. S. Fisher, ibid. 20, L997 (1987); D. S. Fisher and D. A. Huse, Phys. Rev. B 38, 386 (1988).

${ }^{19}$ J. M. Kosterlitz and D. J. Thouless, J. Phys. C 6, 1181 (1973); J. M. Kosterlitz, ibid. 7, 1046 (1974); see also J. F. Fernández, M. F. Ferreira, and J. Stankiewicz, Phys. Rev. B 34, 292 (1986); H. G. Evertz and D. P. Landau, ibid. 54, 12302 (1996).

${ }^{20}$ G. Parisi, Phys. Rev. Lett. 43, 1754 (1979); 50, 1946 (1983); for reviews, see M. Mézard, G. Parisi, and M. A. Virasoro, Spin Glass Theory and Beyond (World Scientific, Singapore, 1987); E. Marinari, G. Parisi, and J. J. Ruiz-Lorenzo, in Spin Glasses, edited by K. H. Fischer and J. A. Hertz (Cambridge University Press, Cambridge, 1991).

${ }^{21}$ This is in contrast to the behavior of $S$ for the nearest-neighbor random bond Ising model in $3 \mathrm{D}$, for which $S \simeq 0.05 k_{B}$ at $T=0$. See S. Kirkpatrick, Phys. Rev. B 16, 4630 (1977); A. K. Hartmann, Phys. Rev. E 63, 016106 (2000).

${ }^{22}$ S. F. Edwards and P. W. Anderson, J. Phys. F 5, 965 (1975).

${ }^{23}$ K. Binder and A. P. Young, Rev. Mod. Phys. 58, 801 (1986); H. G. Katzgraber, M. Palassini, and A. P. Young, Phys. Rev. B 63, 184422 (2001)

${ }^{24}$ J. F. Fernández and J. J. Alonso, Phys. Rev. B 76, 014403 (2007).

${ }^{25}$ M. Palassini and S. Caracciolo, Phys. Rev. Lett. 82, 5128 (1999); H. G. Ballesteros, A. Cruz, L. A. Fernández, V. MartínMayor, J. Pech, J. J. Ruiz-Lorenzo, A. Tarancón, P. Téllez, C. L. Ullod, and C. Ungil, Phys. Rev. B 62, 14237 (2000); H. G. Katzgraber, M. Körner, and A. P. Young, ibid. 73, 224432 (2006). 